Pacific Journal of Mathematics

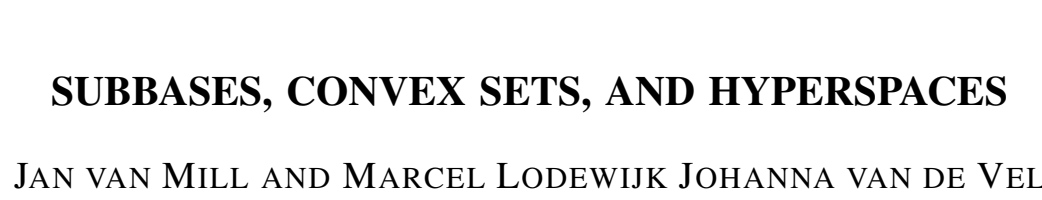




\title{
SUBBASES, CONVEX SETS, AND HYPERSPACES
}

\author{
JAN VAN MILL, MARCEL VAN DE VEL
}

\begin{abstract}
We introduce a notion of topological convexity. Main topics are: compactness of convexity structures, continuity of convex closure operators, and characterization of convex sets.
\end{abstract}

1. Closed subbases. It will be assumed that all spaces are $T_{1}$. We agree to use the word "subbase" for "closed subbase".

A subbase $\mathscr{S}$ of a space $X$ is called a $T_{1}$ subbase if for each $S \in \mathscr{S}$ and for each $x \in X-S$ there is an $S^{\prime} \in \mathscr{S}$ with $x \in S^{\prime} \subset X-S$. $\mathscr{S}$ is called a normal subbase if for each pair of sets $S_{1}, S_{2} \in \mathscr{S}$ with $S_{1} \cap S_{2}=\varnothing$ there exist $S_{1}^{\prime}, S_{2}^{\prime} \in \mathscr{S}$ such that

$$
S_{1} \subset S_{1}^{\prime}-S_{2}^{\prime} ; S_{2} \subset S_{2}^{\prime}-S_{1}^{\prime} ; S_{1}^{\prime} \cup S_{2}^{\prime}=X .
$$

$S_{1}^{\prime}$ and $S_{2}^{\prime}$ are then said to separate (or: to screen) $S_{1}$ and $S_{2}$. Finally, $\mathscr{S}$ is called a binary subbase if each linked system $\mathscr{S}^{\prime} \subset \mathscr{S}$ (i.e., a subcollection $\mathscr{S}^{\prime}$ of $\mathscr{S}$ of which any two members meet) satisfies $\cap \mathscr{S}^{\prime} \neq \varnothing$.

It is well-known that a binary subbase is $T_{1}$ (cf. van Mill [9, Lemma 1]), that a space carrying a binary subbase is compact (use Alexander's lemma), and that a space is completely regular iff it admits a normal $T_{1}$ (sub)base (cf. Frink [6, Thm. 1]; de Groot and Aarts [1, Thm. 2]).

2. Topological convexity structures. The analysis of convex sets in Euclidean space has led to the introduction of axiomatic convexity theory. One of the main purposes of this theory is to investigate in an abstract setting the relationship between various convexity invariants, modelled after famous theorems of Caratheodory, Helly and Radon on convex sets of $\boldsymbol{R}^{n}$.

In this paper we will study convexity structures compatible with a topological structure. Our purpose is not to study the above mentioned invariants, as we do not expect the introduction of a topology to give rise to new relationships in general. Instead, we are mainly concerned with the interaction between the two structures. Some of the main results are summed up at the end of this section. More results and applications can be found in [13], [14], [15], and [18].

In [7, p. 471], Kay and Womble define a convexity (structure) as a pair $(X, \mathscr{C})$, where $\mathscr{C}$ is a collection of subsets of $X$, such that $\varnothing, X \in \mathscr{C}$ and $\cap \mathscr{C}^{\prime} \in \mathscr{C}$ for each nonempty family $\mathscr{C}^{\prime} \subset \mathscr{C}$. $\mathscr{C}$ is also called a convexity structure for (on) $X$, and the members of $\mathscr{C}$ 
are said to be the convex sets of the structure, or $\mathscr{C}$-convex sets. For our purposes, it is more convenient to omit the empty set, and consequently, to require $\cap \mathscr{C}^{\prime} \in \mathscr{C}$ only when $\cap \mathscr{C}^{\prime} \neq \varnothing$.

If a convexity $\mathscr{C}$ is to be compatible with the topological structure of $X$ then it is natural to require that the members of $\mathscr{C}$ be closed, and in fact that $\mathscr{C}$ be a subbase, so that there are sufficiently many convex sets. This leads to the following definition:

Definition 2.1. A topological convexity structure (briefly: a convexity) is a pair $(X, \mathscr{C})$, where $X$ is a topological space, and $\mathscr{C}$ is both a convexity structure on the underlying set of $X$ and a subbase for the topology of $X$. As most spaces considered below will be compact, the latter condition is not very restrictive.

This notion of "subbase convexity" arose from investigations on the Lefschetz fixed point property of superextensions (van de Vel [17]), and it yields an appropriate background for so-called interval structures (van Mill and Schrijver [12]) and for subbasic closure operations on supercompact spaces (van Douwen and van Mill [3]):

Definition 2.2. Let $(X, \mathscr{C})$ be a topological convexity structure, and let $A \subset X$ be a nonempty (closed) set. The convex closure of $A$ relative to $\mathscr{C}$ is defined to be the set

$$
I_{\mathscr{E}}(A)=\cap\{C \mid A \subset C \in \mathscr{C}\} .
$$

See $[3,2.4]$. If no confusion can arise, we write $I$ for $I_{e}$. In case $A=\left\{x_{1}, x_{2}\right\}$, a two point set, we also write $I\left(x_{1}, x_{2}\right)$ instead of $I\left(\left\{x_{1}, x_{2}\right\}\right)$. This set is called the interval between $x_{1}$ and $x_{2}$, see [12, §2].

Examples 2.3. The above definitions cover two well-known concepts of convexity in topology.

(i) Let $X$ be a totally ordered space. A nonempty closed set of $X$ is order-convex iff it is convex relative to the subbase of all closed segments. An interval in the above sense is an order-theoretic interval.

(ii) Let $X$ be a compact convex subset of a locally convex linear space. Then the collection of all closed linearly convex sets in $X$ forms a subbase. Intervals are simply line-segments. This convexity is normal, as can be seen from a Hahn-Banach theorem (Rudin [16, p. 58]).

In the sequel we shall concentrate on compact spaces and on convexities with an abundancy of convex sets. We make "abundancy" precise by requiring the convexity to be normal. The normality 
condition can be compared with the separation of disjoint convex closed sets by a hyperplane.

For normal binary convexities we shall prove a.o. the following results.

THEOREM 2.4. Let $\mathscr{C}$ be a normal binary convexity structure on the space $X$. Then a nonempty closed set $C \subset X$ is $\mathscr{C}$-convex iff $I,\left(x, x^{\prime}\right) \subset C$ for each $x, x^{\prime} \in X$.

THEOREM 2.5. Let $\mathscr{C}$ be a normal binary convexity structure on $X$. Then each $\mathscr{C}$-convex set is a retract of $X$.

Many other convexity structures satisfy the characterization of convex sets as in 2.4, e.g., linear convexity structures. Theorem 2.5 parallels a well-known retraction property of convex sets in locally convex linear spaces (cf. Dugundji [4, Cor. 4.2]). Proofs are given in $\S 3$, together with some other results.

If $\mathscr{C}$ is a topological convexity on $X$, then the collection $\mathscr{C}$ can be topologized by considering $\mathscr{C}$ as a subspace of $H(X)$, the hyperspace of $X$. It is then natural to ask when $\mathscr{C}$ is closed in $H(X)$, and when the convex closure operator $I_{\mathscr{E}}: H(X) \rightarrow \mathscr{C}$ is continuous. Our main result is that these questions are equivalent for compact $X$ :

THEOREM 2.6. If $X$ is a compact space, and if $\mathscr{C}$ is a normal convexity on $X$, then the following assertions are equivalent:

(i) $\mathscr{C}$ is compact;

(ii) $I_{\mathscr{E}}: H(X) \rightarrow \mathscr{C}$ is continuous;

(iii) $\mathscr{C}$ is a retract of $H(X)$.

For other equivalent conditions, and for a proof of 2.6 , see $\S 4$.

Each subbase $\mathscr{S}$ of a space $X$ generates a topological convexity, namely the family

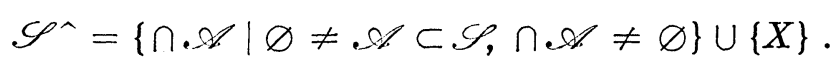

This can be used to produce binary convexities, as follows from our next observation, the simple proof of which is omitted:

FACT 2.7. If $\mathscr{S}$ is a normal binary subbase of $X$, then so is $\mathscr{S}^{\wedge}$. Unfortunately "binary" is essential in the following sense: it need not be true that $\mathscr{S}^{\wedge}$ is normal if $\mathscr{S}$ is a normal subbase for $X$, see 5.3 below.

3. Binary convexity structures. Binary subbases were intro- 
duced by De Groot as a part of (super)compactification theory. They can be interpreted as topological convexity structures of Helly number 2 on compact spaces (the Helly number of a set-theoretic convexity $(X, \mathscr{C})$ is the least natural number $n$ with the property that for each finite $\mathscr{C}^{\prime} \subset \mathscr{C}, \cap \mathscr{C}^{\prime} \neq \varnothing$ whenever each $n$-tuple of members of $\mathscr{C}^{\prime}$ has a nonempty intersection).

As we shall show in $\S 4$, every topological convexity can be "modelled" into a binary one, and this modelling will be used to prove our Theorem 2.6.

We begin with two auxiliary results.

LeMma 3.1. Let $\mathscr{C}$ be a normal binary convexity on $X$, let $A \subset$ $X$ be nonempty and closed, and let $x \in X$. Then

(i) the set $\bigcap_{a \in A} I_{\mathscr{E}}(x, a) \cap I_{\mathscr{B}}(A)$ contains exactly one point;

(ii) if $x \in I_{\mathscr{E}}(A)$, then $\{x\}=\bigcap_{a \in A} I_{\mathscr{E}}(x, a)$.

Proof of (i). The collection

$$
\mathscr{C}^{\prime}=\left\{I_{\mathscr{C}}(x, a) \mid a \in A\right\} \cup\left\{I_{\mathscr{C}}(A)\right\} \subset \mathscr{C}
$$

is obviously linked. Hence, as $\mathscr{C}$ is binary, $\cap \mathscr{C}^{\prime} \neq \varnothing$. Assume that $p \neq q$ are in $\cap \mathscr{C}^{\prime}$. Using the fact that $\mathscr{C}$ is a normal $T_{1}$ subbase and that $X$ is a $T_{1}$ space, we find $C_{0}, C_{1}$ in $\mathscr{C}$ such that

$$
p \in C_{0}-C_{1} ; q \in C_{1}-C_{0} ; \quad C_{0} \cup C_{1}=X .
$$

We may assume that $x \in C_{0}$. If $A \cap C_{0} \neq \varnothing$, then $I_{\mathscr{E}}(x, a) \subset C_{0}$ for some $a \in A$, contradicting that $q \in C_{0}$. Hence, $A \subset C_{1}$, and consequently $I_{\mathscr{Q}}(A) \subset C_{1}$, contradicting that $p \notin C_{1}$.

Proof of (ii). If $x \in I_{\mathscr{E}}(A)$, then $I_{\mathscr{E}}(x, a) \subset I_{\mathscr{E}}(A)$ for each $a \in A$, whence

$$
x \in \bigcap_{a \in A} I_{\mathscr{C}}(x, a)=\bigcap_{a \in A} I_{\mathscr{E}}(x, a) \cap I_{\mathscr{C}}(A) .
$$

The next result is a reformation of [12, Thm. 2.11]:

LeMma 3.2. Let $\mathscr{S}$ be a subbase for the compact space $X$. Then $\mathscr{S}$ is binary iff there is a function

$$
I: X \times X \longrightarrow \mathscr{P}(X) \quad \text { (the power set of } X)
$$

with the following properties:

(i) for each $u, u^{\prime}, x, x^{\prime} \in X: u, u^{\prime} \in I\left(x, x^{\prime}\right)$ iff $I\left(u, u^{\prime}\right) \subset I\left(x, x^{\prime}\right)$;

(ii) for each $x_{1}, x_{2}, x_{3} \in X: I\left(x_{1}, x_{2}\right) \cap I\left(x_{2}, x_{3}\right) \cap I\left(x_{3}, x_{1}\right) \neq \varnothing$;

(iii) for each $S \in \mathscr{S}$ and $x, x^{\prime} \in S, I\left(x, x^{\prime}\right) \subset S$. 
3.3. Proof of Theorem 2.4. Necessity is obvious. To prove sufficiency, let $C_{0} \subset X$ be a nonempty closed set such that $I_{\odot}\left(x, x^{\prime}\right) \subset$ $C_{0}$ for each $x, x^{\prime} \in C_{0}$. Define

$$
I: C_{0} \times C_{0} \longrightarrow \mathscr{P}\left(C_{0}\right)
$$

by $I\left(x, x^{\prime}\right)=I_{\mathscr{E}}\left(x, x^{\prime}\right)$, and let

$$
\mathscr{C} \mid C_{0}=\left\{C \cap C_{0} \mid C \in \mathscr{C}\right\}
$$

Then the subbase $\mathscr{C} \mid C_{0}$ of $C_{0}$ and the map $I$ satisfy the conditions (i), (ii) and (iii) of Lemma 3.2 , proving that $\mathscr{C} \mid C_{0}$ is binary.

Let $p \in I_{\mathscr{C}}\left(C_{0}\right)$, and let

$$
\mathscr{C}^{\prime}=\left\{I_{\mathscr{C}}(p, c) \cap C_{0} \mid c \in C_{0}\right\}
$$

Then $\mathscr{C}^{\prime} \subset \mathscr{C} \mid C_{0}$, and for each pair of points $c_{1}, c_{2} \in C_{0}$,

$$
\varnothing \neq I_{\mathscr{C}}\left(p, c_{1}\right) \cap I_{\mathscr{E}}\left(p, c_{2}\right) \cap I_{\mathscr{O}}\left(c_{1}, c_{2}\right) \subset I_{\mathscr{C}}\left(p, c_{1}\right) \cap I_{\mathscr{C}}\left(p, c_{2}\right) \cap C_{0}
$$

by the binarity of $\mathscr{C}$ and by our assumption on $C_{0}$. This shows that $\mathscr{C}^{\prime}$ is a linked system, whence by the binarity of $\mathscr{C} \mid C_{0}$, $\cap \mathscr{C}^{\prime} \neq \varnothing$. However,

$$
\cap \mathscr{C}^{\prime}=\bigcap_{c \in C} I_{\mathscr{E}}(p, c) \cap C_{0} \subset \bigcap_{c \in C} I(p, c)=\{p\}
$$

by Lemma 3.1(ii), proving that $p \in C_{0}$. It follows that $C_{0}=$ $I_{\mathscr{C}}\left(C_{0}\right) \in \mathscr{C}$.

Another characterization of (normal binary) convexity on superextensions, using partial orderings, can be found in [17, Lemma 2.2].

Our next result is a generalization of [19, III, 2.8], of [11, Thm. 2.2], and of [17, Thm. 2.3]. We need the following notation: Let $H(X)$ be the hyperspace of $X$. If $A_{1}, \cdots, A_{n}$ are nonempty subsets of $X$, then we write

$$
\left\langle A_{1}, \cdots, A_{n}\right\rangle=\left\{C \mid C \in H(X), C \subset \bigcup_{i=i}^{n} A_{i}, C \cap A_{i} \neq \varnothing \text { for } 1 \leqq i \leqq n\right\} .
$$

The topology of $H(X)$ is generated by the open base $\left\{\left\langle 0_{1}, \cdots, 0_{n}\right\rangle \mid n \in N, 0_{1}, 0_{2}, \cdots, 0_{n}\right.$ nonempty and open $\}$, or, equivalently, by the open subbase $\{\langle 0\rangle \mid 0$ nonempty open $\} \cup\{\langle 0, X\rangle \mid 0$ nonempty open $\}$.

3.4. Nearest point mapping theorem. Let $\mathscr{C}$ be a normal binary convexity on $X$. Then for each $x \in X$ and $C \in \mathscr{C}$, the set 


$$
P(x, C)=\bigcap_{0 \in C} I_{\mathscr{E}}\{x, c\} \cap C
$$

contains exactly one point, and the function

$$
p: X \times \mathscr{C} \longrightarrow X,
$$

defined by $\{p(x, C)\}=P(x, C)$, is continuous.

Proof. That $P(x, C)$ is a singleton follows from Lemma 3.1(i) and from $C=I_{\mathscr{C}}(C)$. Let $C \in \mathscr{C}$, and assume $(x, D) \in(X \times \mathscr{C})-p^{-1}(C)$. Then $p(x, D) \notin C$, and since $\mathscr{C}$ is normal $T_{1}$, there exist $C_{0}, C_{1}$, in $\mathscr{C}$ such that

$$
p(x, D) \in C_{0}-C_{1} ; \quad C \subset C_{1}-C_{0} ; \quad C_{0} \cup C_{1}=X .
$$

In particular, $D \cap\left(X-C_{1}\right) \neq \varnothing$ since $p(x, D) \in D-C_{1}$. If $D \not \subset X-C_{1}$, then $x \notin C_{1}$. Indeed, there is a point $d \in D \cap C_{1}$, and $x \in C_{1}$ then implies that

$$
p(x, D) \in I_{6}(x, d) \subset C_{1},
$$

contradicting our assumption. Define

$$
\begin{aligned}
V & =X \times\left(\left\langle X-C_{1}\right\rangle \cap \mathscr{C}\right), & & \text { if } \quad D \subset X-C_{1}
\end{aligned}
$$

Then $V$ is a neighborhood of $(x, D)$ in $X \times \mathscr{C}$ which does not meet $p^{-1}(C)$. For take $\left(x^{\prime}, D^{\prime}\right) \in V$. If $V$ is as in (1), then $D^{\prime} \subset X-C_{1}$, whence

$$
p\left(x^{\prime}, D^{\prime}\right) \in X-C_{1} \subset X-C .
$$

If $V$ is as in (2), then fix $d^{\prime} \in D^{\prime} \cap\left(X-C_{1}\right)$. We find

$$
\left\{d^{\prime}, x^{\prime}\right\} \subset X-C_{1} \subset C_{0},
$$

whence $p\left(x^{\prime}, D^{\prime}\right) \in I_{\mathscr{C}}\left(d^{\prime}, x^{\prime}\right) \subset C_{0} \subset X-C$. This shows that $p^{-1}(C)$ is closed. As $\mathscr{C}$ is a subbase for $X$, it follows that $p$ is continuous.

The mapping $p$ is truly a "nearest point" map: see $[17,2.2,2.3]$ for the behavior of $p$ with respect to certain orderings on superextensions, and [13, Thm. 3.3] for the metric behavior of $p$.

This map has been discovered in various steps, each leading to rather deep consequences. Van Mill used it to prove that a certain subspace of the superextension of $[0,1]$ is homeomorphic to the pseudoboundary of the Hilbert cube, [11, Thm. 3.4], and Van de Vel used it to prove the Lefschetz fixed point property for spaces with binary normal subbases, [17, Cor. 3.7]. 
3.5. Proof of Theorem 2.5. Let $\mathscr{C}$ be a normal binary convexity on $X$, and let $C \in \mathscr{C}$. Then then the restriction

$$
p(-, C): X \longrightarrow X
$$

of the nearest map $p$ obviously retracts $X$ onto $C$.

Let $\mathscr{C}$ be a topological convexity structure on the space $X$. We let $L(X, \mathscr{C})$ denote the subspace of the hyperspace $H(\mathscr{C})$, consisting of all linked systems $\mathscr{L} \subset \mathscr{C}$ which are closed and nonempty.

THEOREM 3.6. Let $\mathscr{C}$ be a normal binary convexity on $X$. Then the intersection operator

$$
L(X, \mathscr{C}) \longrightarrow \mathscr{C},
$$

sending $\mathscr{L} \in L(X, \mathscr{C})$ to $\cap \mathscr{L}$, is continuous.

Proof. $X$ is compact and completely regular (see $\S 1$ ), and hence normal. Notice that $\cap \mathscr{L} \neq \varnothing$ for each $\mathscr{L} \in L(X, C)$ by binarity. Fix a nonempty open set $0 \subset X$, and let $\mathscr{L} \in L(X, \mathscr{S})$. We have to consider two cases for a continuity proof:

(i) $\cap \mathscr{L} \subset 0 . \quad X$ being compact, there exist $L_{1}, \cdots, L_{n} \in \mathscr{L}$ such that $\bigcap_{i=1}^{n} L_{i} \subset 0$. By the normality of $X$, there exist open sets $0_{1}, \cdots, 0_{n}$ such that $L_{i} \subset 0_{i}(1 \leqq i \leqq n)$ and $\bigcap_{i=1}^{n} 0_{i} \subset 0$. Then

$$
V=\left\langle\left\langle 0_{1}\right\rangle \cap \mathscr{C}, \cdots,\left\langle 0_{n}\right\rangle \cap C\right\rangle \cap L(X, \mathscr{C})
$$

is a neighborhood of $\mathscr{L}$ which is easily seen to be mapped into $\langle 0\rangle \cap \mathscr{C}$.

(ii) $\cap \mathscr{L} \cap 0 \neq \varnothing$. Fix a point $x \in \cap \mathscr{L} \cap 0$. As $\mathscr{C}$ is a normal $T_{1}$ subbase, closed under (nonempty) intersections, there exists a $C \in \mathscr{C}$ such that

$$
x \in \operatorname{int} C \subset C \subset 0 \text {. }
$$

Then the set

$$
V=\langle\langle C, X\rangle \cap \mathscr{C}\rangle \cap L(X, \mathscr{C})
$$

is a neighborhood of $\mathscr{L}$, and $V$ is mapped into $\langle 0, X\rangle \cap \mathscr{C}$. Indeed, if $\mathscr{L}^{\prime} \in V$ then $L^{\prime} \cap C \neq \varnothing$ for each $L^{\prime} \in \mathscr{L}^{\prime}$, whence $\mathscr{L}^{\prime} \cup\{C\}$ is a linked system. $\mathscr{C}$ being binary,

$$
\varnothing \neq \cap \mathscr{L}^{\prime} \cap C \subset 0,
$$

proving that $\cap \mathscr{L}^{\prime} \in\langle 0, X\rangle \cap \mathscr{C}$.

Continuity of $A \cap B$ in the variables $A$ and $B$ (or even in one 
variable at the time) seems to be fairly exceptional, as can be seen from the next example using only "nice" sets:

Let $X$ be the unit 2-cell, regarded as a subset of the complex numbers. For each $t \in[0, \pi]$ we let $F_{t} \in H(X)$ denote the line-segment joining 0 with $e^{i t}$. The resulting mapping $F:[0, \pi] \rightarrow H(X)$, sending $t$ to $F_{t}$, is obviously continuous. The map

$$
G: F([0, \pi]) \longrightarrow H(X),
$$

assigning to $F_{t}$ the set $F_{\pi} \cap F_{t}$, is not continuous, since the image of $G \circ F$ consists of the two points $\{0\}$ and $F_{\pi}$ of $H(X)$.

We shall now work towards the continuity of the convex closure operator for normal binary convexities. A lemma is needed:

Lemma 3.7. Let $\mathscr{C}$ be a normal binary convexity for $X$, and let $n \geqq 2$. For each $(n+1)$-tuple $\left(x, x_{1}, \cdots, x_{n}\right) \in X^{n+1}$ the set

$$
F\left(x, x_{1}, \cdots, x_{n}\right)=\bigcap_{i=1}^{n} I_{\mathscr{C}}\left(x, x_{i}\right) \cap I_{\mathscr{E}}\left(x_{1}, \cdots, x_{n}\right)
$$

contains exactly one point, and the function

$$
f: X^{n+1} \longrightarrow X \text {, }
$$

defined by $\left\{f\left(x, x_{1}, \cdots, x_{n}\right)\right\}=F\left(x, x_{1}, \cdots, x_{n}\right)$, is continuous.

Proof. The first part of the statement follows from Lemma 3.1 (i). To prove continuity, let $C \in \mathscr{C}$ and let $\left(x, x_{1}, \cdots, x_{n}\right) \in X^{n+1}-$ $f^{-1}(C)$. Then

$$
\bigcap_{i=1}^{n} I_{\mathscr{C}}\left(x, x_{i}\right) \cap I_{\mathscr{\ell}}\left(x_{1}, \cdots, x_{n}\right) \cap C=\varnothing,
$$

and by the binarity of $\mathscr{C}$, we have either that $I_{\mathscr{E}}\left(x, x_{i}\right) \cap C=\varnothing$ for some $i \in\{1, \cdots, n\}$, or that $I_{\mathscr{C}}\left(x_{1}, \cdots, x_{n}\right) \cap C=\varnothing$. In the first case, it follows from the normality of $\mathscr{C}$ that there is a $C_{0} \in \mathscr{C}$ with

$$
I\left(x, x_{i}\right) \subset \operatorname{int} C_{0} \subset C_{0} \subset X-C .
$$

Let $\pi_{j}: X^{n+1} \rightarrow X$ denote the projection onto the $j$ th-coordinate $(j=$ $0, \cdots, n)$. Then

$$
0=\pi_{0}^{-1}\left(\operatorname{int} C_{0}\right) \cap \pi_{i}^{-1}\left(\operatorname{int} C_{0}\right)
$$

is a neighborhood of $\left(x, x_{1}, \cdots, x_{n}\right)$ which does not meet $f^{-1}(C)$. For if $\left(y, y_{1}, \cdots, y_{n}\right) \in 0$, then $\left\{y, y_{i}\right\} \subset \operatorname{int} C_{0} \subset C_{0}$, whence

$$
f\left(y, y_{1}, \cdots, y_{n}\right) \in I_{\mathscr{E}}\left(y, y_{i}\right) \subset C_{0} \subset X-C .
$$

In the second case, one can proceed in the same way. First, choose $C_{0} \in \mathscr{C}$ such that 


$$
I_{\mathscr{E}}\left(x_{1}, \cdots, x_{n}\right) \subset \operatorname{int} C_{0} \subset C_{0} \subset X-C .
$$

Then put

$$
0=\bigcap_{i=1}^{n} \pi_{i}^{-1}\left(\operatorname{int} C_{0}\right)
$$

This set is a neighborhood of $\left(x, x_{1}, \cdots, x_{n}\right)$ not meeting $f^{-1}(C)$.

THEOREM 3.8. Let $\mathscr{C}$ be a normal binary convexity on $X$. Then the convex closure map

$$
I_{\mathscr{E}}: H(X) \longrightarrow \mathscr{C}
$$

is a retraction of $H(X)$ onto $\mathscr{C}$.

Proof. We use the open subbase of $\mathscr{C} \subset H(X)$, induced by the sets of type $\langle 0\rangle$ or $\langle 0, X\rangle$, where 0 is open in $X$. Fix an open set $0 \subset X$, and assume that $I_{\mathscr{E}}(A) \subset 0$ for a given $A \in H(X)$. As $X$ is compact, and as $\mathscr{C}$ is a normal subbase which is closed under intersection, there exist $C_{1}, \cdots, C_{n} \in \mathscr{C}$ such that

$$
X-0 \subset \bigcup_{i=1}^{n} C_{i} \subset X-I_{\mathscr{E}}(A) \text {. }
$$

$\mathscr{C}$ being normal, there exist $D_{i}, D_{1}^{\prime} \in \mathscr{C}$ with

$$
C_{i} \subset D_{1}^{\prime}-D_{i} ; \quad I_{\mathscr{E}}(A) \subset D_{i}-D_{1}^{\prime} ; \quad D_{i} \cup D_{1}^{\prime}=X(1 \leqq i \leqq n)
$$

Let $D=\bigcap_{i=1}^{n} D_{i}$. Then $D \in \mathscr{C}$ and

$$
I_{\mathscr{E}}(A) \subset \operatorname{int} D \subset D \subset \bigcap_{i=1}^{n}\left(X-C_{i}\right) \subset 0 .
$$

For each $A^{\prime} \in\langle$ int $D\rangle$, we then have $I_{\mathscr{E}}\left(A^{\prime}\right) \subset D \subset 0$.

Assume next that $I_{\mathscr{C}}(A) \cap 0 \neq \varnothing$, and fix a point $p \in I_{\mathscr{8}}(A) \cap 0$. By Lemma 3.1 (ii)

$$
\{p\}=\bigcap_{a \in A} I_{\mathscr{E}}(a, p) \subset 0 .
$$

As $X$ is compact, and as 0 is open, there exist already finitely many $a_{i} \in A(1 \leqq i \leqq n)$ such that

$$
\bigcap_{i=1}^{n} I_{\mathscr{Q}}\left(a_{i}, p\right) \subset 0 .
$$

Consequently, with the notation of Lemma 3.7,

$$
\left\{f\left(p, a_{1}, \cdots, a_{n}\right)\right\}=\bigcap_{i=1}^{n} I_{\mathscr{E}}\left(a_{i}, p\right) \cap I_{\mathscr{B}}\left(a_{1}, \cdots, a_{n}\right) \subset 0 .
$$


By the continuity of $f$ there exist open neighborhoods $V_{i}$ of $a_{i}$, $1 \leqq i \leqq n$, such that for each $n$-tuple $\left(a_{1}^{\prime}, \cdots, a_{n}^{\prime}\right) \in \prod_{i=1}^{n} V_{i}$,

$$
f\left(p, a_{1}^{\prime}, \cdots, a_{n}^{\prime}\right) \in 0 .
$$

The set $\left\langle V_{1}, \cdots, V_{n}, X\right\rangle$ is a neighborhood of $A \in H(X)$, which we now show to be mapped into $\langle 0, X\rangle$. Let $A^{\prime} \in\left\langle V_{1}, \cdots, V_{n}, X\right\rangle$ and choose $a_{i}^{\prime} \in A^{\prime} \cap V_{i}, 1 \leqq i \leqq n$.

Then

$$
\varnothing \neq \bigcap_{i=1}^{n} I\left(a_{i}^{\prime}, p\right) \cap I_{\mathscr{E}}\left(a_{1}^{\prime}, \cdots, a_{n}^{\prime}\right) \subset 0 \cap I_{\mathscr{E}}\left(A^{\prime}\right),
$$

since $\left\{a_{1}^{\prime}, \cdots, a_{n}^{\prime}\right\} \subset A^{\prime}$, and $\left(a_{1}^{\prime}, \cdots, a_{n}^{\prime}\right) \in \prod_{i=1}^{n} V_{i}$. In particular, $0 \cap$ $I_{\mathscr{E}}\left(A^{\prime}\right) \neq \varnothing$. This proves the continuity of $I_{\mathscr{C}} . \quad I_{\mathscr{C}}$ is then a retraction, since $I_{\mathscr{C}}(C)=C$ for each $C \in \mathscr{C}$.

Theorem 3.6 may suggest another way to prove the continuity of convex closure: if $\mathscr{C}$ is a normal binary convexity on $X$, then $I_{\mathscr{6}}$ equals the composition

$$
H(X) \stackrel{F}{\longrightarrow} L(X, \mathscr{C}) \stackrel{G}{\longrightarrow} \mathscr{C},
$$

where

$$
\begin{array}{ll}
F(A)=\{C \mid A \subset C \in \mathscr{C}\} & (A \in H(X)), \\
G(\mathscr{L})=\cap \mathscr{L} & (\mathscr{L} \in L(X, \mathscr{C})) .
\end{array}
$$

Difficulties arise when proving the continuity of $F$ : there is no nice open or closed subbase available for $L(X, \mathscr{C})$.

4. Compact convexity structures. As a consequence of Theorem 3.8, a normal binary convexity $\mathscr{C}$ on a space $X$ is a compact subspace of $H(X)$. The class of such "compact" convexities appears to be considerably larger than the rather restrictive class of normal binary convexities: it contains for instance the linear convexities on compact convex sets in locally convex linear spaces (cf. §5).

We begin with modelling subbases (or convexities) into binary ones.

4.1. Superextensions. Let $\mathscr{S}$ be a closed subbase of $X$. Then $\lambda(X, \mathscr{S})$ denotes the set of all maximal linked systems in $\mathscr{S}$ (see $\S 1$ ), equipped with the topology generated by the closed subbase

$$
\mathscr{S}^{+}=\left\{S^{+} \mid S \in \mathscr{S}\right\}
$$

where $S^{+}=\{\mathscr{L} \mid \mathscr{L} \in \lambda(X, \mathscr{S}), S \in \mathscr{L}\} . \quad$ The resulting space is called the superextension of $X$ relative to $\mathscr{S}_{\text {. }}$. The subbase $\mathscr{S}^{+}$is automatically binary. It is easy to see that for each $S_{1}, S_{2} \in \mathscr{S}$ : 


$$
\begin{array}{lll}
S_{1} \cup S_{2}=X & \text { iff } & S_{1}^{+} \cup S_{2}^{+}=\lambda(X, \mathscr{S}) ; \\
S_{1} \cap S_{2}=\varnothing & \text { iff } & S_{1}^{+} \cap S_{2}^{+}=\varnothing ; \\
S_{1} \subset S_{2} & \text { iff } & S_{1}^{+} \subset S_{2}^{+} .
\end{array}
$$

In this way $\mathscr{S}^{+}$can be regarded as a kind of a model of $\mathscr{S}$. The above equivalences imply that $\mathscr{S}$ is normal iff $\mathscr{S}^{+}$is normal. Being a Wallman-type extension, the space $\lambda(X, \mathscr{S})$ is automatically $T_{1}$. Normality of $\mathscr{S}\left(\mathscr{S}^{+}\right)$implies that $\lambda(X, \mathscr{S})$ is Hausdorff.

If $X$ is a $\left(T_{1}\right)$ space with a $T_{1}$ subbase $\mathscr{S}$, then there is an embedding $i: X \rightarrow \lambda(X, \mathscr{S})$ defined by

$$
i(x)=\{S \mid x \in S \in \mathscr{S}\}, \quad x \in X .
$$

In the sequel, we shall identify $X$ with the subspace $i(X)$ of $\lambda(X, \mathscr{S})$.

For detailed information on superextension theory, the reader can consult Verbeek [19]. A simple example that $\lambda(X, \mathscr{S}) \neq \lambda\left(X, \mathscr{S}^{\wedge}\right)$ can be found in $\left[9\right.$, p. 13]. It is very obvious that $\mathscr{S}^{+} \neq\left(\mathscr{S}^{+}\right)^{\wedge}$ in general.

We now come to a proof of Theorem 2.6. For proof-technical reasons, we give an extended formulation of 2.6 , including three more equivalent conditions.

THEOREM 4.2. Let $X$ be a compact space, and let $\mathscr{C}$ be a normal convexity on $X$. Then the following conditions are equivalent.

(i) $\mathscr{C}$ is compact;

(ii) $I_{\mathscr{E}}: H(X) \rightarrow \mathscr{C}$ is continuous;

(iii) $\mathscr{C}$ is a retract of $H(X)$;

(iv) the mapping $\mathscr{C} \rightarrow \mathscr{C}^{+}$, sending $C \in \mathscr{C}$ to $C^{+} \in \mathscr{C}^{+}$, is a homeomorphism;

(v) the collection

$$
\{\langle C\rangle \cap \mathscr{C} \mid C \in \mathscr{C}\} \cup\{\langle C, X\rangle \cap \mathscr{C} \mid C \in \mathscr{C}\}
$$

is a normal $T_{1}$ subbase for the space $\mathscr{C}$;

(vi) the restriction of $I_{\mathscr{C}}$ to the space of all finite subsets of $X$ is continuous, and a nonempty closed set $A \subset X$ is $\mathscr{C}$-convex iff for each finite $F \subset A, I_{\mathscr{B}}(F) \subset A$.

The implications (ii) $\Rightarrow$ (iii) $\Rightarrow$ (i) are obvious, since $H(X)$ is compact (cf. Michael [8, Thm. 4.2]). We shall prove the following statements: (i) $\Rightarrow$ (ii) and (iv); (iv) $\Rightarrow$ (i) $\Rightarrow$ (v) $\Rightarrow$ (iv); (i) $\Leftrightarrow$ (vi).

Proof of (i) $\Rightarrow$ (ii) and (iv). Recall that $X$ is regarded as a subspace of $\lambda(X, \mathscr{C})$, which at present is closed by the compactness of $X$. Let $g$ denote the restriction of $I_{\mathscr{E}+}$ to $H(X) \subset H(\lambda(X, \mathscr{C}))$, 
and let $h$ denote the restriction of $g$ to $\mathscr{C} \subset H(X)$. The functions $g$ and $h$ are continuous by Theorem 3.8. For each $A \in H(X)$ we have

$$
I_{\mathscr{C}}+(A)=\cap\left\{C^{+} \mid C \in \mathscr{C}, A \subset C^{+}\right\}=\cap\left\{C^{+} \mid A \subset C \in \mathscr{C}\right\},
$$

and it easily follows that

$$
I_{\mathscr{C}+}(A)=\left(I_{\mathscr{E}}(A)\right)^{+} .
$$

This shows that $g(H(X))=h(\mathscr{C}) \subset \mathscr{C}^{+}$.

Regarding $h$ as a map $\mathscr{C} \rightarrow \mathscr{C}^{+}$, one can easily see that this map has a two-sided inverse, which is the mapping $\mathscr{C}^{+} \rightarrow \mathscr{C}$ sending $C^{+}$to $C=C^{+} \cap X$. As $\mathscr{C}$ is compact and Hausdorff, it follows that $h: \mathscr{C} \rightarrow \mathscr{C}^{+}$is a homeomorphism. We then obtain a well-defined continuous mapping

$$
h^{-1} g: H(X) \longrightarrow \mathscr{C}^{+} \longrightarrow \mathscr{C},
$$

which equals $I_{\mathscr{E}}$.

Proof of (iv) $\Rightarrow$ (i). Assume that $\mathscr{C}$ and $\mathscr{C}^{+}$are homeomorphic spaces. We show that $\mathscr{C}^{+}$is a closed subset of $\left(\mathscr{C}^{+}\right)^{\wedge}$. The latter being compact, we then find that $\mathscr{C}^{+}$, and hence also $\mathscr{C}$, is compact. Let $A \in\left(\mathscr{C}^{+}\right)^{\wedge}-\mathscr{C}^{+}$. If $A \cap X=\varnothing$, then $\langle\lambda(X, \mathscr{C})-X\rangle$ is a neighborhood of $A$ not meeting $\mathscr{C}^{+}$(since each $C^{+} \in \mathscr{C}^{+}$satisfies $\left.C^{+} \cap X=C \neq \varnothing\right)$. So assume that $A \cap X \neq \varnothing$, and let $\mathscr{A} \subset \mathscr{C}^{+}$be such that $A=\cap \mathscr{A}$. Then

$$
A \cap X=\cap\left\{C^{+} \mid C^{+} \in \mathscr{A}\right\} \cap X=\cap\left\{C \mid C^{+} \in \mathscr{A}\right\} \in \mathscr{C} .
$$

Hence we can consider $(A \cap X)^{+}$. As we noticed in the proof of (i) $\Rightarrow$ (ii) and (iv), $(A \cap X)^{+}=I_{\mathscr{C}^{+}}(A \cap X)$, whence by the convexity of $A \subset \lambda(X, \mathscr{C}),(A \cap X)^{+} \subset A$. As $A \notin \mathscr{C}^{+}$, we find that $(A \cap X)^{+} \neq$ $A$, and hence there must be a maximal linked system

$$
\mathscr{L} \in A-(A \cap X)^{+} \text {. }
$$

Fix $L \in \mathscr{L} \subset \mathscr{C}$, such that $L \cap(A \cap X)=\varnothing$. $\mathscr{C}$ being normal, we can find $C_{0}, C_{1} \in \mathscr{C}$ such that

$$
L \subset X-C_{0} ; \quad A \cap X \subset X-C_{1} ; \quad\left(X-C_{0}\right) \cap\left(X-C_{1}\right)=\varnothing .
$$

Observe that $\mathscr{L} \in L^{+} \cap A$, whence $L^{+} \cap A \neq \varnothing$, and that

$$
L^{+} \subset \lambda(X, \mathscr{C})-C_{0}^{+}
$$

as one can easily see from $L \subset X-C_{0}$. Hence, the collection

$$
V=\left\langle\lambda(X, \mathscr{C})-C_{0}^{+}, \lambda(X, \mathscr{C})\right\rangle \cap\left\langle\lambda(X, \mathscr{C})-C_{1}^{+}, \lambda(X, \mathscr{C})-X\right\rangle
$$


is a neighborhood of $A$. We show that $V \cap \mathscr{C}^{+}=\varnothing$. Indeed, assume that $D^{+} \in V \cap \mathscr{C}^{+}$. We find that

$$
\begin{aligned}
& D=D^{+} \cap X \subset\left(\left(\lambda(X, \mathscr{C})-C_{1}^{+}\right) \cup(\lambda(X, \mathscr{C})-X)\right) \cap X=X-C_{1}, \\
& \varnothing \neq D^{+} \cap\left(\lambda(X, \mathscr{C})-C_{0}^{+}\right), \text {whence } \varnothing \neq D \cap\left(X-C_{0}\right) .
\end{aligned}
$$

This contradicts with the fact that $X-C_{0}$ and $X-C_{1}$ are disjoint.

Proof of $(\mathrm{i}) \Rightarrow(\mathrm{v})$. First notice that for each $C \in \mathscr{C}$,

$$
\begin{aligned}
& H(X)-\langle C\rangle=\langle X-C, X\rangle ; \\
& H(X)-\langle C, X\rangle=\langle X-C\rangle,
\end{aligned}
$$

and hence that the sets $\langle C\rangle \cap \mathscr{C},\langle C, X\rangle \cap \mathscr{C}$ are closed in $\mathscr{C}$. Assume that $\mathscr{C}$ is compact, let $\mathscr{B} \subset \mathscr{C}$ be closed, and let $D \in \mathscr{C}-\mathscr{B}$. For each $B \in \mathscr{B}$ we have either that $B \not \subset D$, or that $D \not \subset B$.

If $B \not \subset D$, then choose $x \in B-D$, and fix $C_{B}, C_{D} \in \mathscr{C}$ such that

$$
x \in C_{B}-C_{D} ; \quad B \subset C_{D}-C_{B} ; \quad C_{D} \cup C_{B}=X .
$$

In particular, $x \in B \cap \operatorname{int} C_{B}$, and it follows that $\left\langle C_{B}, X\right\rangle$ is a neighborhood of $B$ which does not contain $D$.

If $D \not \subset B$, then choose $y \in D-B$, and fix $C_{B}, C_{D} \in \mathscr{C}$ such that

$$
y \in C_{D}-C_{B} ; \quad B \subset C_{B}-C_{D} ; \quad C_{D} \cup C_{B}=X .
$$

We then find that $\left\langle C_{B}\right\rangle$ is a neighborhood of $B$, and that $D \notin\left\langle C_{B}\right\rangle$. By the compactness of $\mathscr{B}$, a finite number of the selected neighborhoods of type $\left\langle C_{B}, X\right\rangle$ or $\left\langle C_{B}\right\rangle$ suffices to cover $\mathscr{B}$. It easily follows that the sets of type $\langle C\rangle \cap \mathscr{C}$ or $\langle C, X\rangle \cap \mathscr{C}, C \in \mathscr{C}$, form a subbase for the space $\mathscr{C}$.

The proof that this subbase is normal and $T_{1}$ is a routine argument, involving the corresponding assumptions on $\mathscr{C}$. We leave this to the interested reader.

Proof of (i) $\Rightarrow$ (vi). We only have to show that if $A \in H(X)$ is such that for each finite $F \subset A, I_{\mathscr{E}}(F) \subset A$, then $A \in \mathscr{C}$. Let $\left\langle 0_{1}, \cdots, 0_{n}\right\rangle$ be a basic open neighborhood of $A$. Fix $a_{i} \in A \cap 0_{i}, 1 \leqq i \leqq n$, and let $F=\left\{a_{1}, \cdots, a_{n}\right\}$. By assumption,

$$
I_{\mathscr{C}}(F) \subset A \subset \bigcup_{i=1}^{n} 0_{i} ; \quad I_{\mathscr{C}}(F) \cap 0_{i} \neq \varnothing \quad \text { for } \quad 1 \leqq i \leqq n,
$$

and hence $\left\langle 0_{1}, \cdots, 0_{n}\right\rangle$ meets $\mathscr{C}$. It follows that

$$
A \in \text { closure of } \mathscr{C}=\mathscr{C} \text {. }
$$

Proof of (vi) $\Rightarrow$ (i). Let $A \in H(X)-\mathscr{C}$. By assumption, there 
is a finite set $F=\left\{a_{1}, \cdots, a_{n}\right\} \subset A$ and a point $x \in I_{\mathscr{E}}(F)-A$. By the regularity of $X$, there exist open sets $0, P \subset X$ such that $x \in P, A \subset 0$, and $0 \cap P=\varnothing$. Since $I_{\mathscr{C}}$ is continuous on the space of finite sets in $X$, there exist open sets $0_{i} \subset 0$ such that $a_{i} \in 0_{i}(1 \leqq i \leqq n)$ and such that for each $n$-tuple $\left(a_{1}^{\prime}, \cdots, a_{n}^{\prime}\right) \in \prod_{i=1}^{n} 0_{i}, I_{\varepsilon}\left(a_{1}^{\prime}, \cdots, a_{n}^{\prime}\right) \cap P \neq \varnothing$. The set $V=\left\langle 0,0_{1}, \cdots, 0_{n}\right\rangle$ is a neighborhood of $A$. We show that it does not meet $\mathscr{C}$ : let $B \in V$, and fix $b_{i} \in B \cap 0_{i}, 1 \leqq i \leqq n$. We find that

$$
I_{\mathscr{E}}\left(b_{1}, \cdots, b_{n}\right) \cap P \neq \varnothing ; \quad B \subset \bigcup_{i=1}^{n} 0_{i} \cup 0=0 .
$$

It follows that $I_{\mathscr{E}}\left(b_{1}, \cdots, b_{n}\right) \not \subset B$, whence $B \notin \mathscr{C}$.

4.3. Comments. Except for the rather surprising equivalence between compactness of $\mathscr{C}$ and continuity of $I_{\mathscr{C}}$, two other equivalent conditions seem to be interesting: (iv) and (vi). As for (iv), we noticed already that the modelling of $\mathscr{C}$ into a binary $\mathscr{C}^{+}$is combinatorically faithful. Compact convexities are characterized by the fact that this modelling is also topologically faithful.

On the other hand, condition (vi) states that compact convexities satisfy a slightly weaker form of a characterization of convex sets when compared with binary convexities (cf. Theorem 2.6).

The condition ( $v$ ) appears to be rather technical, though it has proved useful already in various applications ([14, Thm. 2.1.] and [18, Thms. 8, 9 and 10]).

5. Some examples and corollaries. Theorem 4.2 shows that a subbase which

(a) is normal and $T_{1}$;

(b) is closed under (nonempty) intersection;

(c) is compact as a hyperspace of convex sets;

must satisfy quite stringent properties. The most interesting types of examples are the normal binary subbases (including Example 2.3(i)), and the ones described in 5.1 below.

EXAMPLE 5.1. Let $X$ be a compact convex subspace of a locally convex linear space, equipped with the subbase (convexity) $\mathscr{C}$ of all closed and linearly convex subsets of $X$ (cf. 2.3(ii)). By Hahn-Banach's theorem ([16, p. 58]) $\mathscr{C}$ is also normal. This convexity is compact, as one can see from an obvious argument on line segments, using the continuity of the algebraic operations.

It follows from Theorem 4.2 that the hyperspace of all closed and linearly convex sets of $X$ is a retract of $H(X)$, with a retraction equal to the convex closure operator. 
EXAMPLE 5.2. Let $X$ be a locally connected continuum, and let $C(X)$ denote the hyperspace of all subcontinua of $X$. Then $C(X)$ is easily seen to be a normal $T_{1}$ subbase for $X$, and $C(X)$ is compact. $C(X)$ is a convexity if and only if $X$ is hereditarily unicoherent. A locally connected hereditarily unicoherent continuum is a tree (cf. Whyburn [21, Thm. 9.1]; a tree is a continuum in which every two distinct points can be separated by a third point, cf. Ward [20, p. 992]). In this case, $C(X)$ is even a binary subbase, as can be derived from the proof of [12, Thm. 4.3]. A direct proof of this fact can be obtained from Lemma 3.2 (which was also taken from [12]): using unicoherence, one can find for each $a, b \in X$ a smallest continuum $I(a, b)$ with $a, b \in I(a, b)$. It is now easy to see that the resulting map $I: X^{2} \rightarrow \mathscr{P}(X)$ and the subbase $C(X)$ satisfy the assumptions (i) (ii) and (iii) of Lemma 3.2, whence $C(X)$ is binary.

In [2, Thm. 2], Curtis and Schori have shown that $C(X)$ is a Hilbert Cube factor (that is, a space whose product with the Hilbert Cube is homeomorphic to the Hilbert Cube), iff $X$ is a Peano continuum. In particular, $C(X)$ is then a retract of $H(X)$. Theorem 4.2 (or 3.8) implies that for the class of dendra (metrizable trees) such a retraction can be well described as the convex closure operator relative to $C(X)$.

ExAmples 5.3. Let $S^{1}$ denote the unit circle. The following collections are easily seen to be closed subbases for $S^{1}(0<r \leqq 2 \pi)$ :

$$
\begin{aligned}
& \mathscr{S}_{r}=\left\{C \in C\left(S^{1}\right) \mid \text { arc length of } C \leqq r\right\} \text {, } \\
& \mathscr{S}_{r}^{\prime}=\left\{C \in C\left(S^{1}\right) \mid \text { arc length of } C<r\right\} \text {. }
\end{aligned}
$$

There is a well-known homeomorphism $h: C\left(S^{1}\right) \rightarrow E^{2}$ (the unit 2-cell) constructed as follows: $h\left(S^{1}\right)=0$, and for each $C \in C\left(S^{1}\right)-\left\{S^{1}\right\}, h(C)$ is the point of $E^{2}$ on the line segment joining 0 with the middle point of the $\operatorname{arc} C$ on a distance

$$
1-\frac{1}{2 \pi}(\operatorname{arc} \text { lenght of } C)
$$

of the origin. Applying this map to the subspaces $\mathscr{S}_{r}, \mathscr{S}_{r}^{\prime}$ of $C\left(S^{1}\right)$, it is easy to see that each $\mathscr{S}_{r}$ is compact and each $\mathscr{S}_{r}^{\prime}$ is noncompact. $\mathscr{S}_{r}$ (resp. $\mathscr{S}_{r}^{\prime}$ ) is closed under intersection iff $r<\pi$ (resp. iff $r \leqq \pi$ ). $\mathscr{S}_{r}$ is nonnormal for each $r<2 \pi$, and $\mathscr{S}_{r}^{\prime}$ is normal iff $r>\pi$.

None of the above subbases therefore satisfies (a), (b), and (c) simultaneously. Notice that $\mathscr{S}_{r}$ and $\mathscr{S}_{r}^{\prime}$ are even binary if $r<2 \pi / 3$.

Let $\mathscr{S}=\mathscr{S}_{r}^{\prime}$ with $r=2 \pi / 3$. Then $\mathscr{S}$ is a normal nonbinary subbase for the circle. The members

$$
A=\{(0,1),(0,-1)\}, \quad B=\{(1,0),(-1,0)\}
$$


of the generated convexity $\mathscr{S}^{\wedge}$ can only be screened by disconnected closed sets. One easily sees that disconnected members of $\mathscr{S}^{\wedge}$ have measure less than $\pi$. Hence $\mathscr{S}^{\wedge}$ is not normal.

We now present some corollaries of the results obtained in $\S \S 3$ and 4 .

CoRollary 5.4. Let $\mathscr{C}$ be a normal binary convexity on $X$. Then the space $\mathscr{C}$ has a normal binary convexity.

Proof. By Theorems 3.8 and $4.2, \mathscr{C}$ admits a normal $T_{1}$ subbase consisting of all sets of type $\langle C\rangle \cap \mathscr{C}$ or $\langle C, X\rangle \cap \mathscr{C}$, where $C \in \mathscr{C}$. This subbase is binary: let the collection

$$
\mathscr{L}=\left\{\left\langle C_{i}\right\rangle \cap \mathscr{C} \mid i \in I\right\} \cup\left\{\left\langle D_{j}, X\right\rangle \cap \mathscr{C} \mid j \in J\right\}
$$

be linked, where $C_{i}, D_{j} \in \mathscr{C}$ for each $i \in I$ and $j \in J$. Choose

$$
\begin{array}{ll}
C_{i i^{\prime}} \in\left\langle C_{i}\right\rangle \cap\left\langle C_{i^{\prime}}\right\rangle \cap \mathscr{C} & i, i^{\prime} \in I, \\
D_{i j} \in\left\langle C_{i}\right\rangle \cap\left\langle D_{j}, X\right\rangle \cap \mathscr{C} & i \in I, j \in J .
\end{array}
$$

Hence

$$
\begin{aligned}
& \varnothing \neq C_{i i^{\prime}} \subset C_{i} \cap C_{i}, \\
& D_{i j} \subset C_{i} ; \quad D_{i j} \cap D_{j} \neq \varnothing,
\end{aligned}
$$

proving that for each $j \in J$ the collection

$$
\left\{C_{i} \mid i \in I\right\} \cup\left\{D_{j}\right\} \subset \mathscr{C}
$$

is linked. Choose

$$
x_{j} \in \bigcap_{i \in I} C_{i} \cap D_{j}, \quad j \in J,
$$

and let $A=I_{\mathcal{S}}\left(\left\{x_{j} \mid j \in J\right\}\right)$. Then $A \subset \bigcap_{i \in I} C_{i}$, and $A \cap D_{j} \neq \varnothing$ for each $j \in J$. Consequently, $\cap \mathscr{L} \neq \varnothing$.

COROLlaRY 5.5. Let $X$ be a continuum with a normal binary convexity $\mathscr{C}$. Then

(i) the space $\mathscr{C}$ has the fixed point property for continuous maps;

(ii) if $X$ is metrizable, then $\mathscr{C}$ is a metric AR.

Proof. The space $H(X)$ is connected, and hence its retract $\mathscr{C}$ is connected. A connected space carrying a normal binary subbase is an acyclic Lefschetz space (cf. van de Vel [17, Cor. 3.7]). If $X$ is in addition metrizable, then $\mathscr{C} \subset H(X)$ is metrizable. It then follows from a result of van Mill ([10, Thm. 4]) that $\mathscr{C}$ is a metric AR. $\square$ 
Statement (ii) can also be derived as follows. A continuum $X$ carrying a normal binary subbase is locally connected (cf. Verbeek [19, Thm. III, 4.1]). If $X$ is metrizable, then $H(X)$ is an AR (cf. Wojdyslawski $[22$, p. 247]), and hence $\mathscr{C}$ is an $\mathrm{AR}$, being a retract of an AR.

5.6. Construction of hyperspaces which are AR's. By the above cited result of Wojdyslawski, the hyperspace of a Peano continuum is an AR. In case a metric continuum is not locally connected, the techniques discussed in the present paper provide a way to construct hyperspaces which are AR's and which are close to the original hyperspace.

Let $\mathscr{S}$ be a normal $T_{1}$ subbase for the metric continuum $X$. Then $\lambda(X, \mathscr{S})$ is Peanian ([19, Cor. IV 2.4, Thm. III 4.1]), and consequently

$\left(\mathscr{S}^{+}\right)^{\wedge}$ is an $\mathrm{AR}$, being a retract of an $\mathrm{AR}$ (Theorem 3.8).

By a recent result of Edwards (cf. [5]) every (compact metric) $\mathrm{AR}$ is a Hilbert cube factor. It would be desirable to find conditions on $(X, \mathscr{S})$ such that $\left(\mathscr{S}^{+}\right)^{\wedge}$ is not only a Hilbert cube factor but is homeomorphic to the Hilbert cube itself.

Added in proof. (1) The equivalence of (i) and (ii) in Theorem 4.2 has been obtained more economically by R. E. Jamison in his 1974 dissertation (University of Washington, Seattle). Rather unfortunately, no part of this dissertation has been published, so that the authors became aware of it only recently.

(2) In [18] a notion of "convexity" has been developed for nonclosed sets, and which is based on the above studied type of topological convexity structure. If one adds the nonclosed "convex" sets to the given convexity structure, one obtains the "topological alignments" which were studied by R. E. Jamison. In this way, both approaches are basically the same, and a unifying account is given in a forthcoming paper of the second author, entitled: "Pseudoboundaries and pseudo-interiors for topological convexities".

(3) In another forthcoming paper of the second author, entitled: "Finite dimensional convexity structures II: the invariants", it will be shown that the "classical" invariants of a convexity are rather deeply affected by certain topological conditions, e.g., concerning dimension. This seemingly contradicts with the expectation raised in $\S 2$ above. We emphasize, however, that these results only work under rather stringent (though natural) restrictions such as connectedness of convex sets. 


\section{REFERENCES}

1. J. de Groot, J. M. Aarts, Complete regularity as a separation axiom, Canad. J. Math., 21 (1969), 96-105.

2. D. W. Curtis and R. M. Schori, $2^{X}$ and $C(X)$ are homeomorphic to the Hilbert cube, Bull. Amer. Math. Soc., 80 (1974), 927-931.

3. E. K. van Douwen, J. van Mill, Supercompact spaces, to appear in General Topology and Appl.

4. J. Dugundji, An extension of Tietze's theorem, Pacific J. Math., 1 (1951), 353-367.

5. D. Edwards, in preparation.

6. O. Frink, Compactifications and semi-normal spaces, Amer. J. Math., 86 (1964), 602-607.

7. D. C. Kay, E. W. Womble, Axiomatic convexity theory and relationships between the Caratheodory, Helly, and Radon numbers, Pacific J. Math., 38 (1971), 471-485.

8. E. Michael, Topologies on spaces of subsets, Trans. Amer. Math. Soc., 71 (1951), 152-182.

9. J. van Mill, On supercompactness and superextensions, Report 37, Vrije Universiteit Amsterdam.

10. The superextension of the closed unit interval is homeomorphic to the Hilbert cube, Fund. Math., 103 (1978), 151-175.

11. — A pseudo-interior of $\lambda I$, Comp. Math., 36 (1978), 57-82.

12. J. van Mill and A. Schrijver, Subbase characterizations of compact topological spaces, General Topology and Appl., 10 (1979), 183-201.

13. J. van Mill and $\mathrm{M}$. van de Vel, Convexity preserving mappings in subbase convexity theory, Proc. Kon. Ned. Acad. Wetensch., ser. A 81 (1) (1978), 76-90.

14. - On superextensions and hyperspaces, Topological Structures II, Math. Centre Tract 115, Mathematisch Centrum, Amsterdam, (1979), 169-180.

15. J. van Mill and E. Wattel, An external characterization of spaces which admit binary normal subbases, Amer. J. Math., 100 (1978), 987-994.

16. W. Rudin, Functional Analysis, McGraw-Hill, N.Y., 1973.

17. M. van de Vel, Superextensions and Lefschetz fixed point structures, Fund. Math., 104 (1978), 33-48.

18. — A Hahn-Banach theorem in subbase convexity theory, in Canad. J. Math., 32 (4) (1980), 804-820.

19. A. Verbeek, Superextensions of topological spaces, M.C. Tract 41, Amsterdam, 1972.

20. L. E. Ward, A note on dentrites and trees, Proc. Amer. Math. Soc., 5 (1954), 992-994.

21. G. T. Whyburn, Cut points in general topological spaces, Proc. Nat. Acad. Sci., 61 (1968), 380-387.

22. M. Wojdyslawski, Sur la contractilité des hyperespaces de continus localement connexes, Fund. Math., 30 (1938), 247-252.

Received March 18, 1977 and in revised form May 28, 1980.

VRije Universiteit,

Amsterdam, The Netherlands 


\section{PACIFIC JOURNAL OF MATHEMATICS}

\section{EDITORS}

DONALD BABBITT (Managing Editor)

University of California

Los Angeles, CA 90024

HUGo RossI

University of Utah

Salt Lake City, UT 84112

C. C. MOORE and ANDREW OGG

University of California

Berkeley, CA 94720
J. DUGUNDJI

Department of Mathematics

University of Southern California

Los Angeles, CA 90007

R. FinN and J. Milgram

Stanford University

Stanford, CA 94305

\section{ASSOCIATE EDITORS}
R. ARENS
E. F. BECKENBACH
B. H. NeumanN
F. WOLF
K. Yoshida

\section{SUPPORTING INSTITUTIONS}

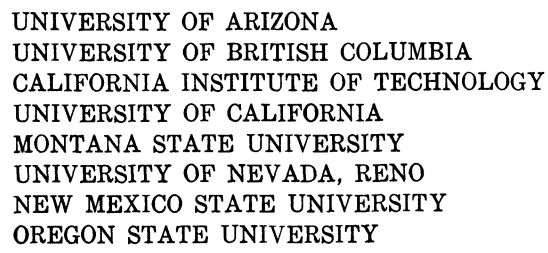

UNIVERSITY OF ARIZONA

UNIVERSITY OF BRITISH COLUMBIA CALIFORNIA INSTITUTE OF TECHNOLOGY

UNIVERSITY OF CALIFORNIA

MONTANA STATE UNIVERSITY

UNIVERSITY OF NEVADA, RENO

NEW MEXICO STATE UNIVERSITY

OREGON STATE UNIVERSITY

\author{
UNIVERSITY OF OREGON \\ UNIVERSITY OF SOUTHERN CALIFORNIA \\ STANFORD UNIVERSITY \\ UNIVERSITY OF HAWAII \\ UNIVERSITY OF TOKYO \\ UNIVERSITY OF UTAH \\ WASHINGTON STATE UNIVERSITY \\ UNIVERSITY OF WASHINGTON
}

The Supporting Institutions listed above contribute to the cost of publication of this Journal, but they are not owners or publishers and have no responsibility for its content or policies.

Mathematical papers intended for publication in the Pacific Journal of Mathematics should be in typed form or offset-reproduced, (not dittoed), double spaced with large margins. Please do not use built up fractions in the text of the manuscript. However, you may use them in the displayed equations. Underline Greek letters in red, German in green, and script in blue. The first paragraph or two must be capable of being used separately as a synopsis of the entire paper. Please propose a heading for the odd numbered pages of less than 35 characters. Manuscripts, in triplicate, may be sent to any one of the editors. Please classify according to the scheme of Math. Reviews, Index to Vol. 39. Supply name and address of author to whom proofs should be sent. All other communications should be addressed to the managing editor, or Elaine Barth, University of California, Los Angeles, California, 90024.

50 reprints to each author are provided free for each article, only if page charges have been substantially paid. Additional copies may be obtained at cost in multiples of 50 .

The Pacific Journal of Mathematics is issued monthly as of January 1966. Regular subscription rate: $\$ 102.00$ a year (6 Vols., 12 issues). Special rate: $\$ 51.00$ a year to individual members of supporting institutions.

Subscriptions, orders for numbers issued in the last three calendar years, and changes of address shoud be sent to Pacific Journal of Mathematics, P.O. Box 969, Carmel Valley, CA 93924, U.S.A Old back numbers obtainable from Kraus Periodicals Co., Route 100, Millwood, NY 10546.

\section{PUBLISHED BY PACIFIC JOURNAL OF MATHEMATICS, A NON-PROFIT CORPORATION}

Printed at Kokusai Bunken Insatsusha (International Academic Printing Co., Ltd.). 8-8, 3-chome, Takadanobaba, Shinjuku-ku, Tokyo 160, Japan. 


\section{Pacific Journal of Mathematics}

Vol. 92, No. $2 \quad$ February, 1981

Bruce Allem Anderson and Philip A. Leonard, Sequencings and Howell designs

Kevin T. Andrews, Representation of compact and weakly compact

operators on the space of Bochner integrable functions . . . . . . . . 257

James Glenn Brookshear, On the structure of hyper-real $z$-ultrafilters . . . . . 269

Frank John Forelli, Jr., A necessary condition on the extreme points of a class of holomorphic functions. II ...................... 277

Richard J. Friedlander, Basil Gordon and Peter Tannenbaum, Partitions of groups and complete mappings ......................... 283

Emden Robert Gansner, Matrix correspondences of plane partitions ......295

David Andrew Gay and William Yslas Vélez, The torsion group of a radical extension ..........................................

André (Piotrowsky) De Korvin and C. E. Roberts, Convergence theorems for some scalar valued integrals when the measure is Nemytskii ...... 329

Takaŝi Kusano and Manabu Naito, Oscillation criteria for general linear ordinary differential equations $\ldots \ldots \ldots \ldots \ldots \ldots \ldots \ldots \ldots \ldots \ldots \ldots \ldots \ldots \ldots \ldots$

Vo Thanh Liem, Homotopy dimension of some orbit spaces .......... 357

Mark Mahowald, $b o$-resolutions . . . . . . . . . . . . . . . . . . . 365

Jan van Mill and Marcel Lodewijk Johanna van de Vel, Subbases, convex

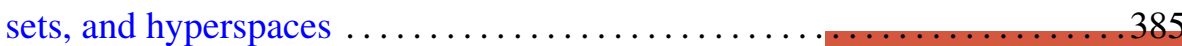

John F. Morrison, Approximations to real algebraic numbers by algebraic numbers of smaller degree $\ldots \ldots \ldots \ldots \ldots \ldots \ldots \ldots \ldots \ldots \ldots \ldots \ldots$

Caroline Series, An application of groupoid cohomology . . . . . . . . . . 415

Peter Frederick Stiller, Monodromy and invariants of elliptic surfaces . . . 433 Akihito Uchiyama, The factorization of $H^{p}$ on the space of homogeneous

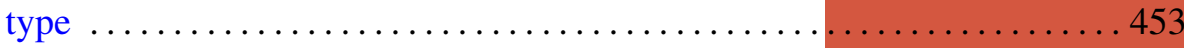

Warren James Wong, Maps on simple algebras preserving zero products.

II. Lie algebras of linear type 